\title{
ADVANCEMENT IN THE MOBILE APP REVIEW SYSTEM TO ENHANCE QUALITY OF MOBILE APPLICATIONS
}

\author{
Sandeep S Machiraju, Anil K Bandi, Ajay K Yaramati, Tulasi R \\ Yadavalli, \& Venkata N Inukollu \\ College of Science and Engineering, University of Houston-Clear Lake, USA
}

\begin{abstract}
The market for mobile applications has enormously increased in the past five years. According to the App Annie reports, the gross annual revenue is projected to exceed $\$ 189$ billion by 2020. Developing and enhancing mobile applications will vastly facilitate in increasing the market for mobile applications. User reviews of mobile apps play a vital role in enhancing the quality of currently existing mobile applications, and, in the development of new high quality mobile applications. Typically, user reviews are either in the form of several lines of text or numerical rating or both. Both forms of rating are meant to aid the prospective users in app installation/purchasing. User reviews also assist the developers in identifying technical glitches (if any) and in providing further advanced updates to the mobile app. The objective of this paper is twofold. Firstly, this paper identifies the currently existing app review systems and analyzes each of these app review systems in detail. Secondly, based on the analysis, we have introduced a four-step app review system that will be instrumental in enhancing the quality of mobile applications. In addition, we have presented the results of a survey (sample size $=100$ ) that was administered to comprehend the effectiveness of the proposed app review system.
\end{abstract}

\section{KEYWORDS}

Mobile App, App Reviews, Mining Reviews, User Rating, User Feedback, User Satisfaction, App Distribution Platforms, IOS \& Android OS

\section{INTRODUCTION}

Mobile applications have significantly increased in number in the past five years and will continue to grow in the coming years [1]. Mobile applications are presently available through various application distribution platforms, such as Apple store, Google Play store, Windows Phone store and many others. These app stores enable users to search, buy and install various software applications. The popularity of mobile apps is growing very rapidly. According to a survey [2], As of March 2017, over 2,800,000 apps are available in the Google play store. The app downloads are anonymous, with around one billion app downloads per month.

Dhinaharan Nagamalai et al. (Eds) : SIGEM, CSEA, Fuzzy, NATL - 2017

pp. 25- 36, 2017. (C) CS \& IT-CSCP 2017

DOI : $10.5121 /$ csit.2017.70903 


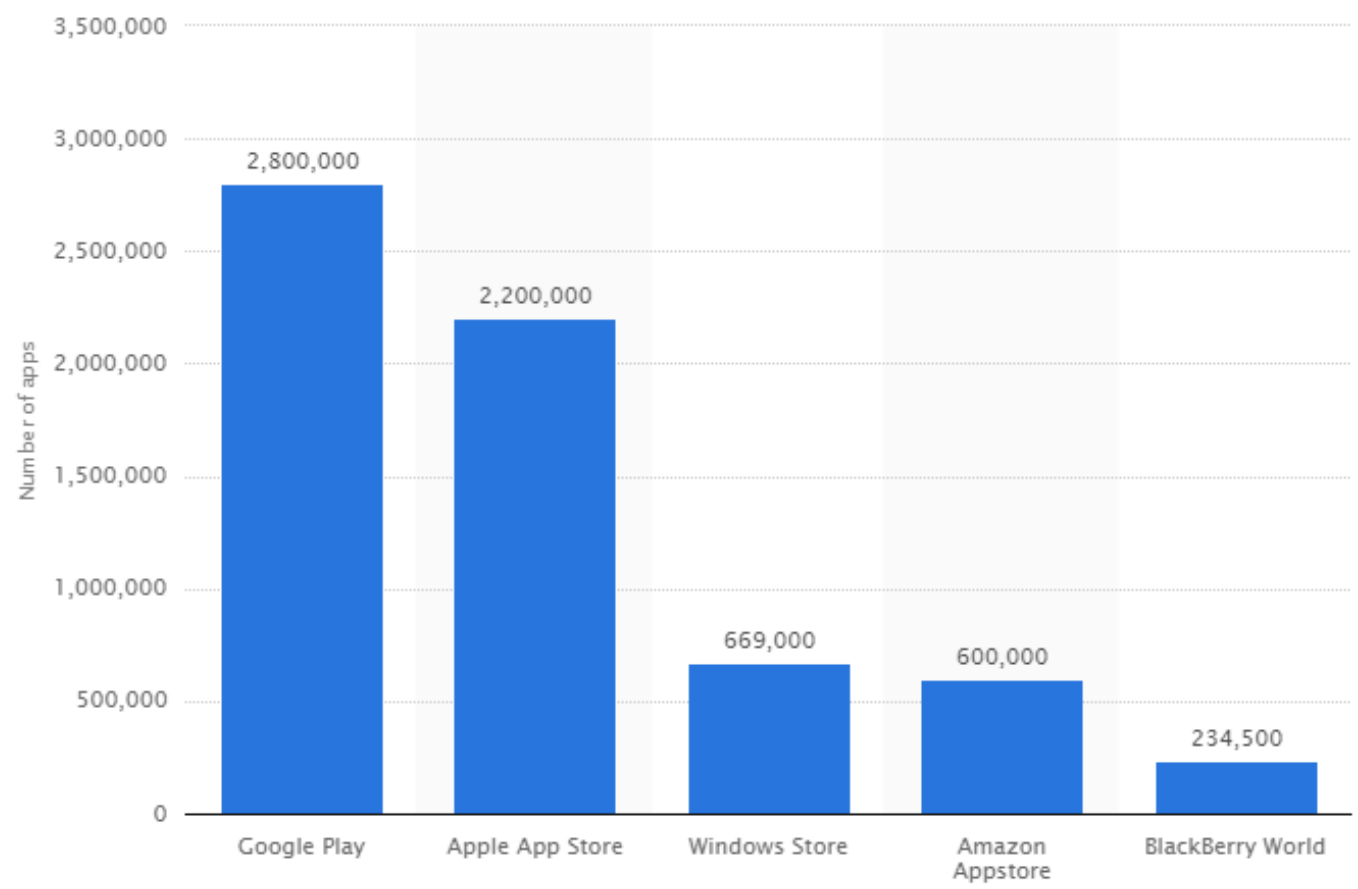

Figure 1: Apps available in various Mobile App Distribution Platforms

Mobile applications have now become a fundamental marketing tool for various businesses. Mobile apps are a smart means to connect to the target audience [3]. Mobile apps aid in: increasing customer engagement, promoting new products and services, online ecommerce transactions, boosting traffic to the products/services and in enhancing end user experience. With the rising popularity of mobile applications, app developers, are, constantly investing a significant amount of time, in gathering and analyzing the user reviews, to, further advance the quality of mobile applications[14]. Several times, the developers experience the absence of effective user reviews. Usually, the user reviews and feedbacks for mobile apps, are, provided by the application distribution platforms, through which the app is distributed. Users who purchase or download an app from a particular platform, can rate and write a review for that app. Ratings and reviews are publicly visible to all the users and developers of that app. Applications with higher ratings will rank higher in the apps list of a distribution platform, which, will increase the visibility of the app to a larger audience. The increased visibility of the app will escalate the number of downloads of the app thereby increasing the revenue. Mobile app reviews primarily consist of the following valuable information: bug reports, issue reports, feature requests among several others [4]. The information gathered from the user reviews is valuable to three stakeholders: App Developers, App Users and App Distribution Platforms.

\section{MOBILE APP USER REVIEWS}

Mobile app - User reviews play a crucial role in the purchasing/installation decisions of users. Positive reviews and high star ratings by the users will empower an app to be ranked in the list of top 100 apps in the distribution platforms. Ranking success will enable an app to be easily discoverable in the app store; thereby increasing the number of downloads of the app and making 
the app successful [13]. The mobile app reviews are useful for three stakeholders: Users, Developers and App Distribution Platforms. After deliberate analysis of the user reviews and its applications, the authors have developed a block diagram depicting the prominence of user reviews for Users, Developers and App Distribution Platforms.

By adapting the approach of the block diagram developed by (Venkata N Inukollu et al, 2017) [5], that depicts the different views of a system, the authors have developed a block diagram that depicts three different views of the mobile app review system: User view, System view and Developer view.

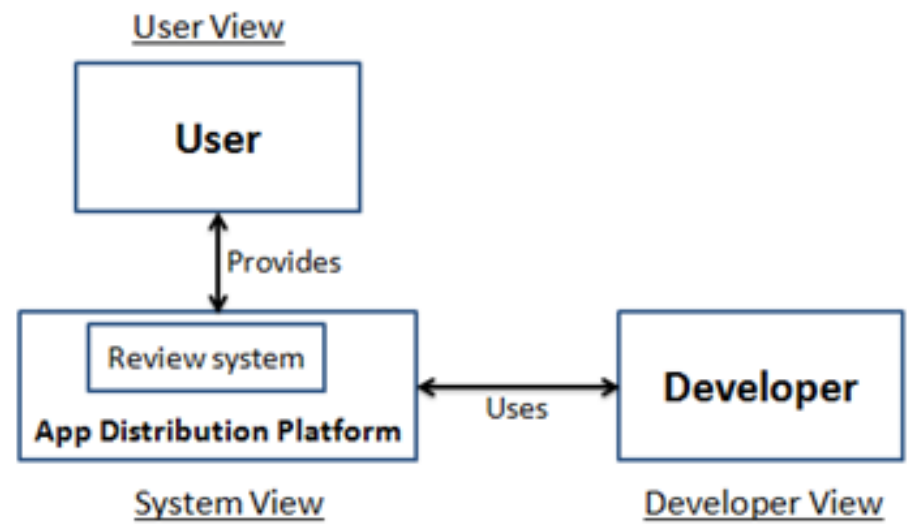

Figure 2: Block Diagram that depicts different views (User, System and Developer view)

- User View: This view describes how effectively and efficiently the users are able to provide the reviews to mobile applications (from this section, we will refer mobile application reviews as app reviews). From the user's point of view, a proficient review system is required by the users to effectively express their views and experiences (positive, negative or neutral) with respect to the mobile application.

- System View: This view describes about the role of the app distribution platforms such as Apple store, Google Play store, Windows Phone store and many others in providing the collection of app reviews to both the users and the developers. From the system point of view, the system should provide an interface that will be helpful: to the users to express their views and to the developers to understand the views expressed by the users with ease. The review system should be robust and truly simple with the end goal that the App Distribution platforms can gather and present the collected reviews to the users and developers with ease.

- Developer view: The developers require an adept platform that will greatly aid in analyzing the requirements and reviews of the users. Understanding the user requirements and reviews will enable the developers in creating innovative applications, and in providing impactful improvements and updates to the existing applications.

Based on the analysis of the roles and requirements of the three stakeholders (classified above), the authors felt the need for an improved review system that will be greatly beneficial both to the users and the developers. 


\section{RELATED WORK}

Understanding user requirements is fundamental for developing superior quality mobile apps and enhancing the quality of currently existing mobile apps. User requirements for mobile applications can be collected in the form of user reviews for the apps. An app is downloaded by several users for versatile purposes. Every user is unique and has a unique perspective, which is applicable even to reviews about mobile applications. Different segments of users, view/download an app for different purposes. For example, students use the apps for education and entertainment purposes, whereas businesses use the apps for promoting/marketing their products and services. Thus, the user segments have been classified as follows: Businesses, Employees/Working Individuals, Students and Researchers. The classified segments can provide feedback related to technical features of an app and also about non-technical features. Technical features include Security, Compatibility, Stability, Accuracy and Bugs among several others whereas; non-technical aspects include Performance, Attractiveness, Costs and features. Currently, the user feedback with respect to different features is analyzed by using the following review systems:

\section{a) Star Rating}

Star rating is one of the most commonly used review system. The star rating generally ranges from 1 to 5. Users' rate the app based on their experience with the app. High star rating indicates positive reviews. Based on the inputs from the users, the average rating is calculated and is displayed as a single number (4.2/5) as shown in figure 3. Along with the star rating, users will also be provided with a text area to express their comments/reviews in the text form.

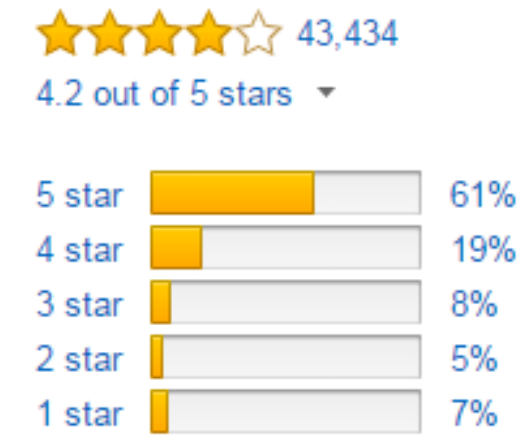

b) Polling

Figure 3: Five Star Rating

The Polling system is one of the most simple review systems. Here the users provide the reviews in a few clicks (depending on the number of questions). The users are prompted for their responses in the form of Yes/no (or) True/false answer format. Based on the responses provided by the users, the results/numbers are calculated and the resultant output is demonstrated as Pie charts, Bar graphs, Percentages and other pictorial forms. User's opinions cannot be justified with the resultant numbers as it is just a Polling system and users do not explain their choices of options or their opinions in any textual form. 


\section{Do you believe in horoscopes?}
Yes
No
Maybe

Vote

Figure 1: Polling System

Do you believe in horoscopes?

ves

Ess

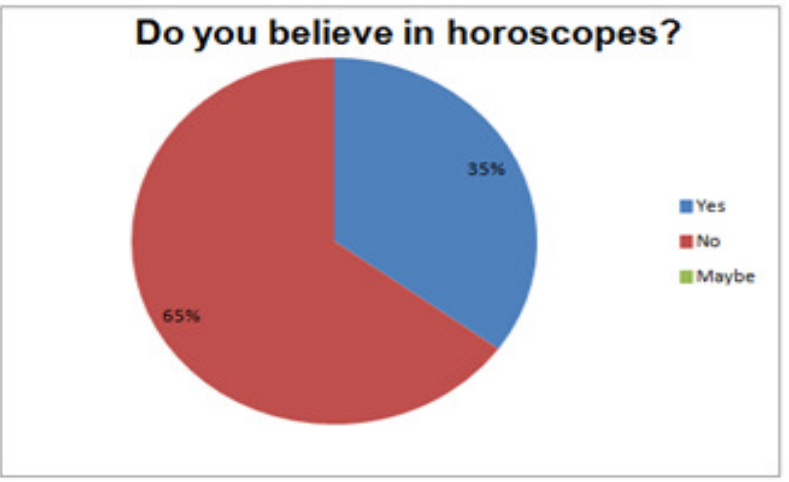

Figure 5: Polling Results

\section{c) Voting}

Voting system is similar to Polling system with few differences. The major difference between Polling and Voting is: Unlike the Polling system where the user has only 2 options (T/F or $\mathrm{Y} / \mathrm{N})$ to choose from, the voting system provides the users with more than two options to choose from. Also, the count for each vote will be displayed in this case.

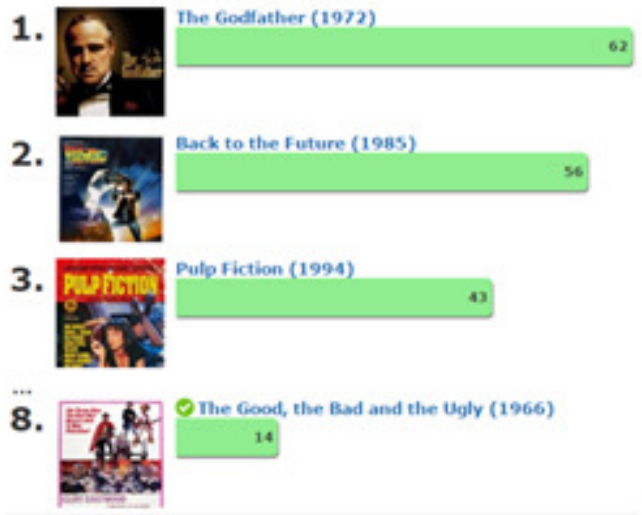

Figure 6 Voting Results 


\section{d) Comment}

Users will be given an option to comment along with their rating. Using this option, users can support/justify their rating by describing few key points as shown below. The comments assist: the developers in providing necessary updates to the apps and the users in installation/ purchasing decisions i.e. whether an app is of their expected standards and is worth downloading/purchasing.

REVIEWS

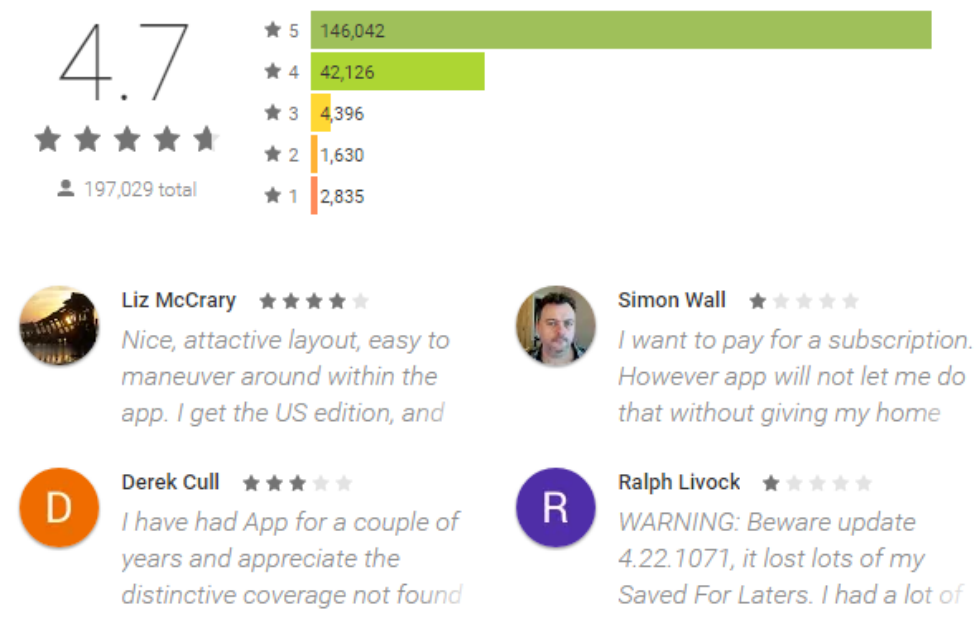

Figure 7: Comment along with 5-Star Rating system

Most of the App Distribution platforms commonly use the 5-star rating system along with the comment system. However, this review system is not comprehensive and robust, and thus, does not provide effective output to the developers and the users. According to the study[6], they analyzed 10k+ mobile apps in Google play store and found an issue related to the current 5-star rating system. They found that the app stores are very resilient — once the app reaches substantial number of ratings, then the Google play store rating system is resilient to fluctuations because the current rating system provides the average of the ratings of the entire life cycle of that app. This provides the inappropriate details to both users and developers. As the current app rating system is five star rating system, it does not depicts the actual satisfaction of user. The current rating system does not provide any facility for the technical people in providing their feedback. These are some of the drawbacks that the current app rating system has. On the other hand, we have external tools, which analyze the data [7] and provide the results to the developers. Also, there are empirical studies on mining and summarizing the user reviews based on various perspectives [8][9]. Phong Minh Vu et al. performed the mining of user reviews by a keywordbased approach and Minqing $\mathrm{Hu}$ et al. worked on the mining and summarizing of customer reviews. The above-mentioned research methods follow an arduous approach and require installation of additional external tools for the purpose of analysis of user reviews. By considering all the limitations, we have proposed a new approach, which helps users in providing as well as developers in getting the appropriate reviews for the mobile app. 


\section{Proposed System}

Considering the limitations of the existing systems in collecting the user reviews, the authors have devised a new and enhanced review system, which will be useful to all the three stakeholders (Users, Developers and Application distribution platforms).

\section{DEVELOPER-CUSTOMER-REVIEW (DCR) CYCLE:}

To achieve an enhanced and superior quality app, it is greatly required to collect the reviews from the users in an effective and proficient format. To accomplish the above-mentioned objective, the authors have introduced the Developer-Customer-Review (DCR) cycle, shown in figure 7.

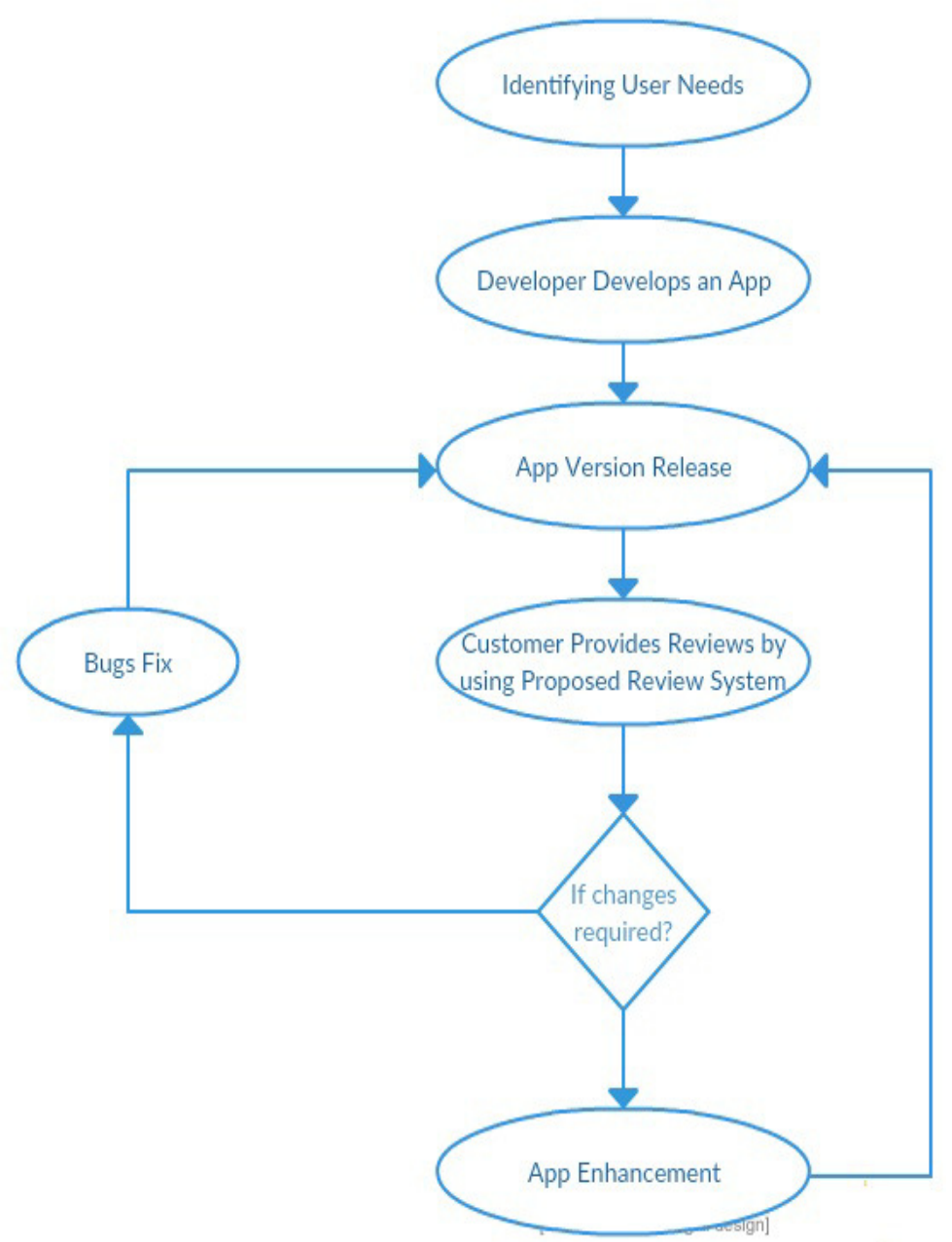

Figure 8: Developer-Customer Cycle (DCR)

In the first step, before the commencement of the actual development, user needs will be identified and gathered. Based on the inputs from the users, the actual development will begin. Once the app is developed, the app will be released as versions. The actual review process starts here. After the collection of various reviews, the developers will be provided with the gathered 
information in different formats. Post analysis of reviews, if any changes are required, the appropriate process will be initiated (Bug fixes or App enhancement).

The proposed review system meets the expectations of both the technical and non-technical user segments, as the system includes both the generalized review and the technical review. Technical savvy users can provide the reviews by choosing the technical terms, and the non-technical users can skip those steps. The process is explained in detail in the column: Steps involved in proposed review system.

The proposed review system is beneficial to both the users and the app developers. Developers can merely look at the reported technical defective factors, and, can rapidly estimate the glitches in the app, and, can very quickly decide on the next move to remove the bugs and to enhance the performance of the app.

In the proposed review system, the user has to complete only four steps to finish the review process, and, thus, is fairly simple and easy to use. A non-technical user does not require reviewing the technical factors and is provided with an optional comment box which can be used to provide user reviews not related to technical factors. A technical user can provide a technical review by choosing out of the 8 relevant technical defective factors including: crashing, compatibility, interface, security, hidden cost, performance, error reports, and resource heavy. The list of the technical defective factors is the resultant of the research conducted by Thung et al. [10] Tian et al. [11], who performed mining of information and categorized the issues into various complaint types. The related information from various research efforts [10][11][12] has greatly helped us in creating the list of different technical defective factors.

\section{STEPS INVOLVED IN PROPOSED REVIEW SYSTEM:}

Step 1:In this step, the system will ask whether the user is willing to provide a review. If the user is interested in providing a review, the user can select the accept button, else, the user can opt for the reject button.

Step 2:The user can rate his level of satisfaction with regards to using the app and the level varies from user to user. In this step, a user can give his satisfaction level on a scale of 1 to 5 . According to [13]: Companies deploying emotional-connection-based strategies, and, metrics to design, prioritize, and measure the customer experience will definitely have increasing number of customers. This particular step of collecting the satisfaction levels, captures, both the positive and negative emotions of the users towards the app. This step will greatly help in recording the feelings and emotions of the users. Gathering and measuring the satisfaction levels of users is only half of the story, and the next half of the story would include: gathering of information regarding the list of technical factors that are not up to the expected standards and thereby have lowered the satisfaction levels of the users.

Step3:In step 2, both the technical and non-technical users provide their reviews based on their overall level of satisfaction with the app. In Step 3, as shown below, the users can select from the list of technical factors that they would like to report. The technical issues most commonly faced by the users are short-listed in this step. Here, non-technical users who are unfamiliar with the technical terms can choose "Others" as an option and can type their comments in the provided comments box (as shown in the below figure). 


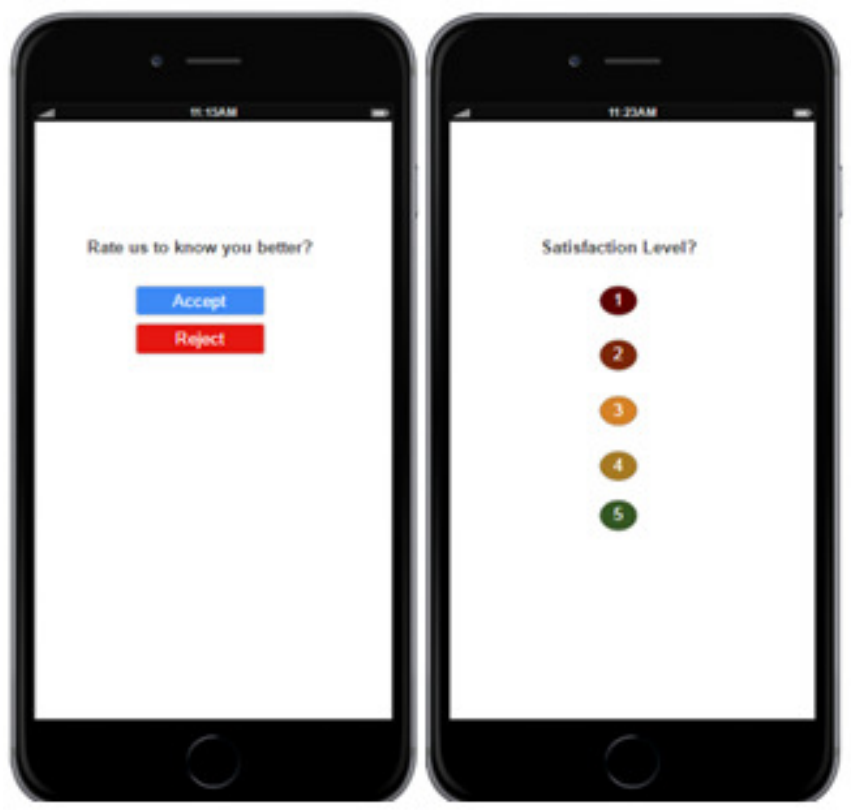

Step $1 \&$ Step 2

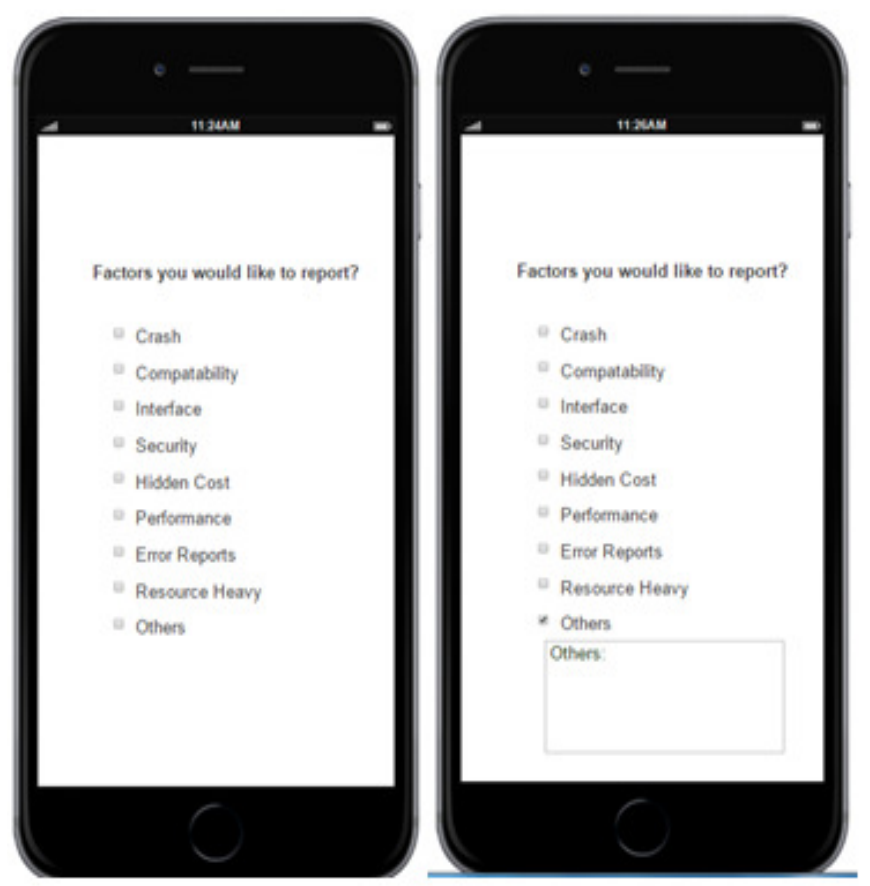

Step 3

\section{Final Output:}

Based on the reviews provided by the users, the output is generated in the form of two graphs that can be comprehended by both technical savvy users and non-technical users. Below shown is a sample of output graphs based on the user reviews. 
For non-technical users, the Satisfaction-level graph will be more comprehensible and helpful in assisting decisions related to downloading or updating the desired app.
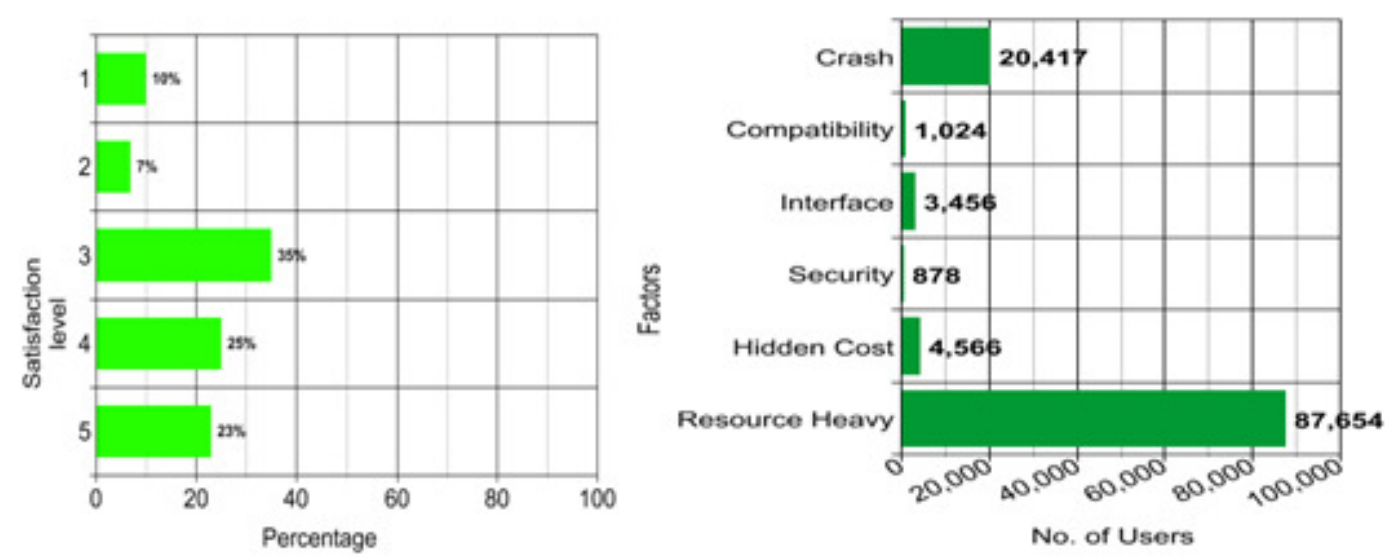

For technical savvy users, the second graph along with the first (Satisfaction level) graph will aid in the comprehension process of the nature and performance of the app. The two graphs combined together will aid the users in arriving at the best decision regarding the installation / updating of an app.

\section{SURVEY RESULTS}

We have conducted a Literature Survey to determine the issues related to the current app review system and to comprehend the effectiveness of the proposed review system in terms of the ease of the users with the proposed review system. We then developed a draft of the survey. A panel of experts assembled and reviewed the draft survey (Panel includes four professors from the University, Software Engineering Department). According to the feedback from the experts, we have modified the survey and finalized. This finalized survey consists of three sections: First section discusses about the Methodology we have chosen for the review system; second section discusses about the User Interface of our proposed review system and final section will includes the questions that will ask the feedback for further improvements of our proposed system. These questions are categorized into three types Agree/Disagree, Yes/No and Feedback.

The survey was conducted with a sample size of 100 students ( 3 sections, each section includes 35 students (approx) and each section at a time) in our university. Before providing the survey handouts, we have presented our topic to the students for better understanding of our concept. Then we distributed the handouts to all the students in the section. After survey was conducted successfully in all the three sections, we have collected the papers and performed analysis on the survey feedback. After the analysis, we got to know that $78 \%$ of the people liked our proposed review system compared with the current app review system. The output was generated in the form of a pie chart, is as shown below. In the below pie chart, the green color demonstrates a positive response and the red color demonstrates a negative response. 


\section{Survey Summary Stats}

\section{Total Hits}

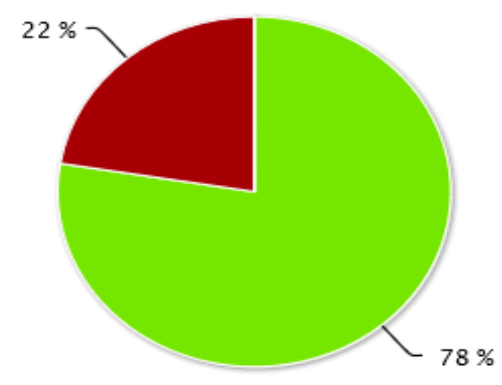

Figure 9. Summary of Survey Results

\section{CONCLUSION \& FUTURE WORK}

In this research effort, we have proposed a theoretical approach that will significantly improve the mobile app review system. All the users (that we have mentioned in section 2) have many advantages by adopting this approach. We have also provided the usability test results by conducting survey. By analyzing the results, it was clearly mentioned that most of the people liked the proposed review system. By considering these results, In future we are going to develop a system that depicts the proposed review system and will conduct usability analysis on the system.

\section{REFERENCES}

[1] Bosomworth, Danyl. "Mobile marketing statistics 2015." Leeds: Smart Insights (Marketing Intelligence) Ltd (2015).

[2] Statista, " App Stores: Number of Apps in Leading App Stores 2017," [Accessed 21 March 2017].

[3] M. Rajput, "How Essential Mobile Apps Are For Business Growth Nowadays"

[4] McIlroy, S., Ali, N., Khalid, H., \& Hassan, A. E. (2016). Analyzing and automatically labelling the types of user issues that are raised in mobile app reviews. Empirical Software Engineering, 21(3), 1067-1106.

[5] Machiraju, S. S., Athukuri, A. K., Gampa, S., Makela, N. B., \& Inukollu, V. N.. "Application Based Smart Optimized Keyboard for Mobile Apps."Computer Science \& Information Technology (CS \& IT), 2017.

[6] Ruiz, Israel J. Mojica, et al. "Examining the Rating System Used in Mobile-App Stores." IEEE Software, 2016.

[7] Jun Lee, S., and Keng S. "A review of data mining techniques." Industrial Management \& Data Systems, 2001. 
[8] Vu, P.M., Nguyen, T.T., Pham, H.V. and Nguyen, T.T., "Mining user opinions in mobile app reviews: A keyword-based approach" Proceedings of the 30th IEEE/ACM International conference, 2015.

[9] Hu, Minqing, and Bing Liu. "Mining and summarizing customer reviews." Proceedings of the tenth ACM SIGKDD international conference on Knowledge discovery and data mining. ACM, 2004.

[10] F. Thung, S. Wang, D. Lo, and L. Jiang, "An empirical study of bugs in machine learning systems," in Proceedings of the 23rd International Symposium on Software Reliability Engineering, 2012, pp. 271-280.

[11] Tian, P. Achananuparp, I. N. Lubis, D. Lo, and E.-P. Lim, "What does software engineering community microblog about?" in Proceedings of the 9th IEEE Working Conference on Mining Software Repositories, 2012, pp. 247-250.

[12] A. Zorfas and D. Leemon, "An Emotional Connection matters more than Customer Satisfaction", [Web]. [Accessed: 29 August 2016].

[13] Sarah, "Developers can finally respond to App Store reviews-here is how it works", [Web]

[14] Fu, Bin, et al. "Why people hate your app: Making sense of user feedback in a mobile app store." Proceedings of the 19th ACM SIGKDD international conference on Knowledge discovery and data mining. ACM, 2013.

[15] Inukollu, Venkata N., Divya D. Keshamoni, Taeghyun Kang, and Manikanta Inukollu. "Factors influencing quality of mobile apps: Role of mobile app development life cycle.", 2014 